\title{
miR-197-3p-induced downregulation of lysine 63 deubiquitinase promotes cell proliferation and inhibits cell apoptosis in lung adenocarcinoma cell lines
}

\author{
YANG CHEN $^{1,2}$ and CHUNLU YANG ${ }^{2}$ \\ ${ }^{1}$ Department of Thoracic Surgery, Chest Hospital of Shenyang, Shenyang, Liaoning 110044; ${ }^{2}$ Department of Thoracic Surgery, \\ The First Affiliated Hospital of China Medical University, Shenyang, Liaoning 110001, P.R. China
}

Received April 19, 2017; Accepted October 24, 2017

DOI: $10.3892 / \mathrm{mmr} .2017 .8333$

\begin{abstract}
Lung adenocarcinoma (LUAD) is a common cause of cancer-associated mortality. The dysregulation of microRNA (miR) expression has been reported to induce lung carcinogenesis. In the present study, miR-197-3p upregulation was detected within LUAD tissues compared with in adjacent noncancerous tissues. The suppression of miR-197-3p expression was confirmed to inhibit proliferative ability and induce apoptosis of LUAD cell lines; miR-197-3p overexpression within the HBE cell line exhibited opposing effects. Via in silico modeling, western blot analyses and dual-luciferase assays, it was confirmed that miR-197-3p directly targets the lysine 63 deubiquitinase (CYLD) gene. In the present study, the expression of miR-197-3p was negatively associated with CYLD mRNA expression within LUAD cell lines. In conclusion, the findings of the present study have provided novel insight into the association of miR-197-3p with LUAD proliferation and apoptotic regulation; the miR-197-3p/CYLD axis may serve as a novel potential therapeutic target for the treatment of LUAD.
\end{abstract}

\section{Introduction}

Lung cancer is one of the main causes of cancer-associated mortality worldwide, based on histological investigations into small cell and non-small cell lung cancer. The latter can be further sorted into several types, among which adenocarcinoma accounts for $\sim 50 \%$ (1). Despite the administration of targeted treatments for lung adenocarcinoma (LUAD), the survival rates associated with this disease have not improved (2). In order to develop novel therapeutic treatments

Correspondence to: Dr Chunlu Yang, Department of Thoracic Surgery, The First Affiliated Hospital of China Medical University, 155 Nanjingbei Street, Shenyang, Liaoning 110001, P.R. China E-mail: clyang@cmu.edu.cn

Key words: microRNA-197-3p, lysine 63 deubiquitinase, lung adenocarcinoma, proliferation, apoptosis for LUAD, further investigation into the molecular mechanism underlying lung carcinogenesis is required.

MicroRNAs (miRNAs/miRs) are molecules 22 nucleotides long that serve important roles in the regulation of target gene expression to subsequently modulate cellular function (3). Irregular miRNA expression has been detected within LUAD samples, which may suggest the interplay of these molecules in the initiation and development of cancer (4). However, further investigation is required to identify which of the aberrantly expressed miRNAs have the potential to serve as therapeutic targets in the development of effective treatment. A recent study reported that miR-197-3p is markedly upregulated within LUAD samples compared with in adjacent noncancerous tissues; as an oncomiR, downregulation of miR-197-3p induces lung cancer apoptosis and inhibits proliferation (5). However, the putative functions and mRNA targets of miR-197-3p have yet to be investigated.

Lysine 63 deubiquitinase (CYLD) is a cytoplasmic protein with three cytoskeletal-associated protein-glycine-conserved domains, which functions as a deubiquitinating enzyme $(6,7)$. A previous study demonstrated that CYLD is downregulated within LUAD tissues compared with in adjacent noncancerous tissues (8); further investigation is required to understand the detailed mechanisms of CYLD dysregulation in LUAD.

In the present study, the expression levels of miR-197-3p within LUAD and adjacent noncancerous tissues were analyzed to investigate the effects of miR-197-3p on cell proliferation and apoptosis. A bioinformatics analysis suggested that CYLD may be a target of miR-197-3p; the interaction between miR-197-3p and CYLD was confirmed via dual-luciferase assays. Additionally, the potential mechanism underlying cell proliferation and apoptosis mediated by miR-197-3p was investigated.

\section{Materials and methods}

Clinical specimen collection, RNA extraction and reverse transcription-quantitative polymerase chain reaction ( $R T-q P C R)$. A total of 32 fresh LUAD tissues (cancer group) and adjacent noncancerous lung tissues $(2 \mathrm{~cm}$ from the margin of resection which had been confirmed as R0 resection; control group) were excised from patients hospitalized at The First Affiliated 
Hospital of China Medical University (Shenyang, China) between August 2015 and August 2016. Patients did not receive chemotherapy or radiotherapy, and tissues were frozen in liquid nitrogen prior to use. The present study was approved by the Human Research Ethics Committee of The First Affiliated Hospital of China Medical University (Shenyang, China; IRB Approval 2012-40-2). Informed consent was obtained from all patients prior to enrolment in the present study.

RNA extraction was performed using the SV Total RNA Isolation system (Promega Corporation, Madison, WI, USA) according to the manufacturer's protocol. RT-qPCR analysis of miR-197-3p was carried out as previously described (9). The mRNA expression levels of CYLD were analyzed by RT-qPCR using SYBR Green qPCR Master Mix (Takara Biotechnology Co., Ltd. Dalian, China) and an ABI 7500 Fast System thermocycler (Applied Biosystems; Thermo Fisher Scientific, Inc., Waltham, MA, USA). Initial denaturation was at $95^{\circ} \mathrm{C}$ for $30 \mathrm{sec}$, followed by annealing at $95^{\circ} \mathrm{C}$ for $5-10 \mathrm{sec}$ and extension at $60^{\circ} \mathrm{C}$ for $30-34 \mathrm{sec}$ for 40 cycles. Protein expression was calculated using the $2^{-\Delta \Delta \mathrm{Cq}}$ method (10) and normalized to U6.

Cell culture. The normal lung tissue cell line HBE and human LUAD cell lines, HCC827 and Calu-3 were obtained from the Cell Bank of the Type Culture Collection of the Chinese Academy of Sciences (Shanghai, China). Cells were cultured in RPMI-1640 medium (Gibco; Thermo Fisher Scientific, Inc.) containing $10 \%$ fetal bovine serum (Gibco; Thermo Fisher Scientific, Inc.), $100 \mathrm{IU} / \mathrm{ml}$ penicillin and $100 \mu \mathrm{g} / \mathrm{ml}$ streptomycin at $37^{\circ} \mathrm{C}$ with $5 \% \mathrm{CO}_{2}$.

Synthetic RNA oligonucleotides and transient transfection. miRNA inhibitors, mimics and a miRNA negative control (NC) were synthesized by Shanghai GenePharma Co., Ltd. (Shanghai, China). The 5'-3' sequences of the three miRNAs were as follows: Mimics, CGGGUAGAGAGG GCAGUGGGAGG and UUCACCACCUUCUCCACCCAG C; inhibitor, AAGUGGUGGAAGAGGUGGGUCG; and NC, CAGUACUUUUGUGUAGUACAA. CYLD overexpression vectors and the NC vector were obtained from Shanghai GeneChem Co., Ltd. (Shanghai, China). Cells (1-1.5x10 $/$ well) in a 6-well plate were transiently transfected with miRNAs $\left(5 \mu \mathrm{g} /\right.$ well) using JetPrime ${ }^{\circledR}$ (Polyplus-transfection SA, Illkirch, France), according to the manufacturer's protocol. Cells were collected for analysis $48 \mathrm{~h}$ post-transfection.

$R N A$ extraction from cell lines and RT-qPCR. Total RNA was extracted from transfected cells using TRIzol (Invitrogen; Thermo Fisher Scientific, Inc.); RT-qPCR analyses of miR-197-3p and CYLD were performed in triplicate, according to the aforementioned protocols. The primer sequences used were as follows: CLYD forward, 5'-TGCCTTCCAACTCTC GTCTTG-3' and reverse, 5'-AATCCGCTCTTCCCAGTA GG-3'; GAPDH forward, 5'-CATGTTCGTCATGGGTGT GAACC-3' and reverse, 5'-GGTCATGAGTCCTTCCACGAT ACC-3'. The mRNA expression levels of miR-197-3p and CYLD within the control group were set to 1 .

Protein extraction and western blot analysis. Following lysis with radioimmunoprecipitation assay buffer (Beyotime
Institute of Biotechnology, Haimen, China), transfected HCC827 cells were harvested and total proteins were extracted with a protein extraction kit (Beyotime Institute of Biotechnology) via centrifugation at $12,000 \times \mathrm{x}, 4^{\circ} \mathrm{C}$ for $20 \mathrm{~min}$. A bicinchoninic acid protein assay kit (Beyotime Institute of Biotechnology) was used to determine protein concentration and $40 \mu \mathrm{g}$ of protein per lane was separated by $10 \%$ SDS-PAGE and subsequently transferred onto a nitrocellulose membrane at a constant electric current of $400 \mathrm{~mA}$ for $1 \mathrm{~h}$. Membranes were blocked for $1 \mathrm{~h}$ at room temperature with 5\% bovine serum albumin (Thermo Fisher Scientific, Inc.) and were incubated with the following primary antibodies: Anti-CYLD (1:2,000; ab137524; Abcam, Cambridge, UK) and anti-GAPDH $\left(1: 2,500 ;\right.$ ab9485; Abcam) overnight at $4^{\circ} \mathrm{C}$. The membranes were then washed four times in tris buffered saline with Tween 20 and were incubated with anti-rabbit horseradish peroxidase-conjugated secondary antibody $(1: 3,000$; ab6721; Abcam) for $30 \mathrm{~min}$ at $37^{\circ} \mathrm{C}$. Proteins were visualized by enhanced chemiluminescence plus western blotting substrate (Thermo Fisher Scientific, Inc.).

Cell proliferation assays. MTT assays were conducted to assess the proliferative ability of transfected HBE, HCC 827 and Calu- 3 cells. A total of $5 \times 10^{3}$ cells/well per cell group were seeded in 96-well plates. At 0, 24, 48 and $72 \mathrm{~h}, 10 \mu 1$ MTT solution was applied to the wells; plates were subsequently incubated at $37^{\circ} \mathrm{C}$ for $3 \mathrm{~h}$. Following MTT incubation, $150 \mu \mathrm{l}$ dimethyl sulfoxide was added to the wells and the plates were agitated at low speed for $10 \mathrm{~min}$. Cell viability was measured at $490 \mathrm{~nm}$ using a microplate reader (Bio-Rad Laboratories, Inc., Hercules, CA, USA).

Caspase-3/7 activity analysis. A total of $3-5 \times 10^{3}$ transfected HCC 827 or Calu-3 cells were seeded in 96-well plates. After 48 h, cells were treated with Caspase-Glo ${ }^{\circledR} 3 / 7$ reagent (Promega Corporation) according to the manufacturer's protocol; following agitation for $30 \mathrm{sec}$, cells were incubated for $2 \mathrm{~h}$ at room temperature. Fluorescence activity was analyzed using a GloMax ${ }^{\circledR} 96$ Microplate Luminometer system (Promega Corporation). Relative fluorescent activity was quantified by setting the blank control to 1 .

miR-197-3p target prediction. To investigate the association between miR-197-3p and cell function, two independent databases, TargetScan (http://www.targetscan.org/vert_71/) and miRanda (http://www.microrna.org/microrna/getGeneForm.do) were employed to predict the targets of miR-197-3p; predicted genes were collated. From the results of cell apoptosis and proliferation assays, CYLD was selected to investigate the effects of miR-197-3p on cell function.

Dual-luciferase assays. To confirm whether miR-197-3p can interact with CYLD mRNA, a Dual-Luciferase miRNA Target Expression system was used. The following reporter plasmids: pGLO-wild-type (wt)-CYLD and pGLO-mutant (mut)-CYLD of miR-197-3p were constructed by Shanghai GeneChem Co., Ltd. There are two regions in pGLO-wt-CYLD that miR-197-2 may bind to. Thus, reporter plasmids containing site 1 and 2 were separately constructed to investigate the effect of each site on CYLD expression. The mutant sequences of the CYLD 

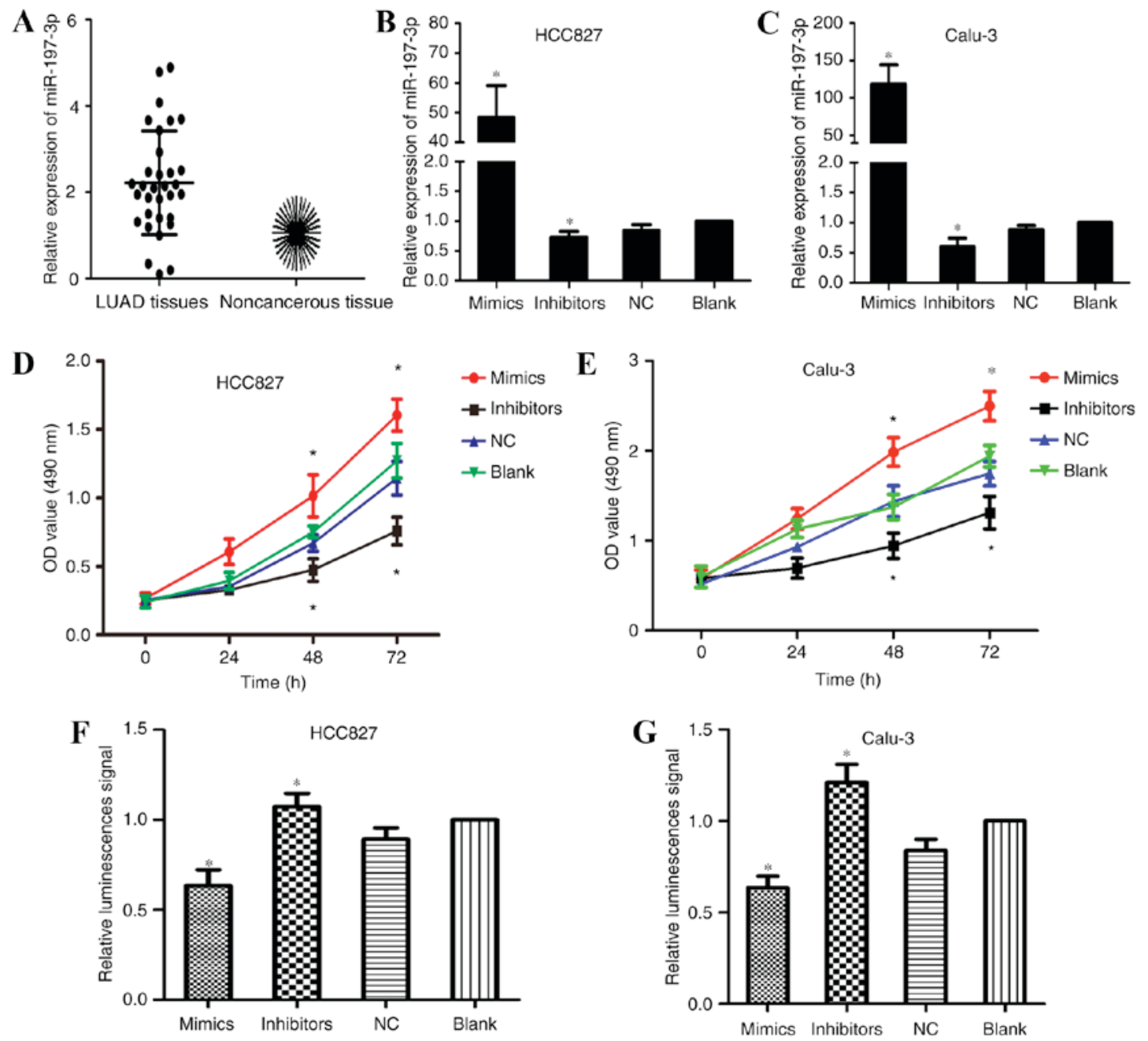

Figure 1. (A) miR-197-3p expression was greatly upregulated within LUAD tissues compared with adjacent noncancerous tissues. The irregular distribution of the expression levels within the LUAD tissues are presented as a vertical scatter plot. The sunflower plot was used to demonstrate the expression levels within the adjacent noncancerous tissues, which served as the control; each petal represents a sample. Reverse transcription-quantitative polymerase chain reaction was applied to confirm transfection efficiency 48 h post-transfection and analyze miR-197-3p expression levels within (B) HCC 827 and (C) Calu-3 cell lines transfected with either miR-197-3p mimics, inhibitors or NC. Proliferative ability of transfected (D) HCC827 and (E) Calu-3 cells was determined via MTT assays; miR-197-3p mimics promoted cell growth compared with in the control group. Caspase-3/7 activation was quantified via relative luminescence signal detection within (F) HCC827 and (G) Calu-3 cells transfected with miR-197-3p mimics, inhibitors or NC. "P<0.05 vs. blank group. LUAD, lung adenocarcinoma; miR, microRNA; NC, negative control; OD, optical density.

miR-197-3p binding region were as follows: Site 1, position 92-114 of CYLD 3'-UTR, 5'-GCAAGTTCTGTCTTTTGT TGTCT-3'; and site 2, position 1469-1491 of CYLD 3'-UTR, 5'-AAGTGCTGTTTTGGGTGTTGTCG-3'. The firefly luciferase reporter plasmids including wild-type or mutant CYLD plasmids and the internal control, Renilla luciferase plasmid pRL-TK (at a ratio of 10:1) were co-transfected with miR-197-3p mimics or NC into HCC 827 cells using JetPRIME ${ }^{\circledR}$. A total of $48 \mathrm{~h}$ following co-transfection, relative luciferase activity compared with Renilla luciferase activity was assessed using the Dual-Luciferase ${ }^{\circledR}$ Reporter Assay system (Promega Corporation) according to the manufacturer's protocol. For comparisons, values for cells with NC + mut-CYLD 3'-UTR group were set equal to 1 .

Statistical analysis. SPSS 23.0 software (IBM Corp., Armonk, NY, USA) and GraphPad Prism 5.0 (GraphPad Software, Inc., La Jolla, CA, USA) were used for all statistical analyses. Data are presented as the mean \pm standard deviation of at least
3 independent experiments. Student's t-test were employed to compare the differences between cancerous and noncancerous tissues. The analysis of variance and Dunnett-t test were used to compare which specific groups were significantly different in cell assays. Spearman's correlation analysis was used to assess the association between miR-197-3p and CYLD mRNA expression. $\mathrm{P}<0.05$ was considered to indicate a statistically significant difference.

\section{Results}

miR-197-3p inhibition suppresses the proliferative ability of HCC 827 and Calu-3 cells. qPCR was employed to detect the expression levels of miR-197-3p in 32 paired samples of LUAD and adjacent noncancerous tissues. The results of the present study demonstrated that miR-197 expression was markedly upregulated within LUAD tissues (Fig. 1A). To investigate the effects of miR-197-3p within LUAD, scrambled miR-197-3p (NC), and miR-197-3p mimics and inhibitors were 
A

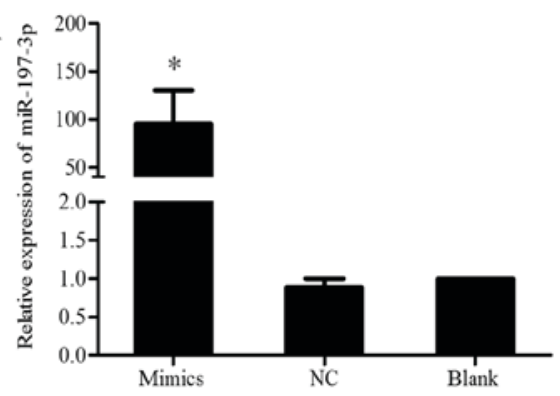

D
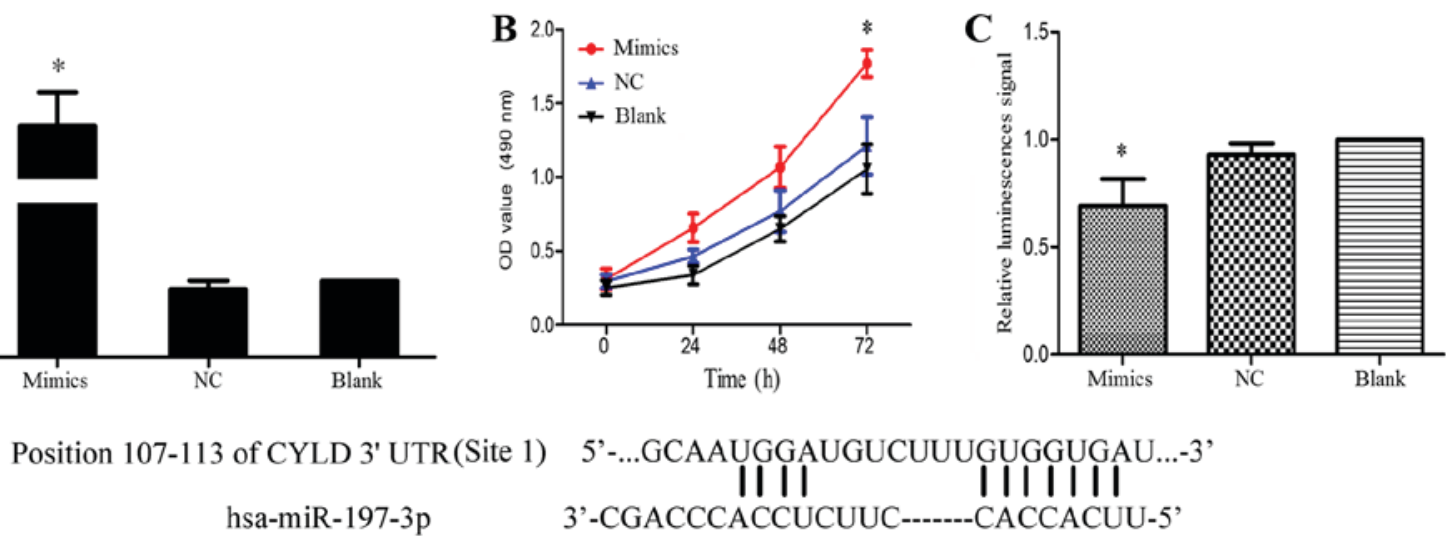

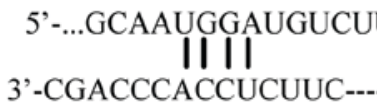

IIIIII

Position 1484-1490 of CYLD 3' UTR (Site 2)

hsa-miR-197-3p

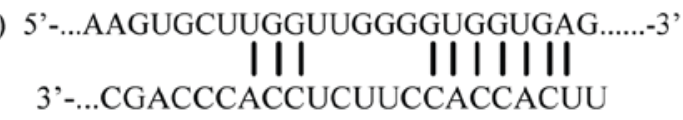

$\mathbf{E}$

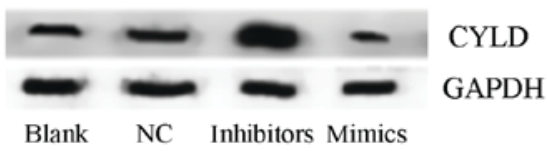

$\mathbf{F}$

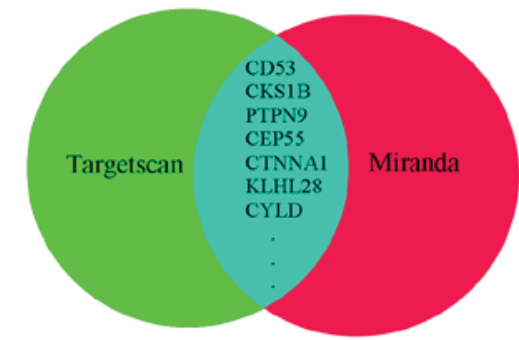

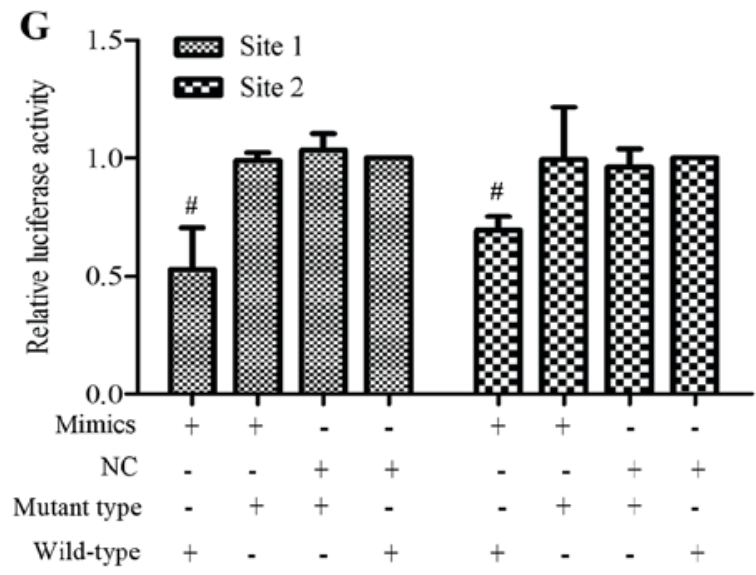

Figure 2. (A) Relative expression levels of miR-197-3p were significantly elevated in the mimics group compared with in the control groups. (B) MTT assay of HBE cells transfected with miR-197-3p mimics. Proliferation was significantly higher in the mimics group. "P<0.05 vs. blank group. (C) Apoptotic analysis of HBE cells transfected with miR-197-3p mimics. Caspase activity was decreased in the mimics group. "P<0.05 vs. blank group. (D) Putative miR-197-3p binding sites in the 3'-UTR of CYLD mRNA. (E) Effects of miR-197-3p mimics and inhibitors on CYLD expression in HCC827 cells, as detected using western blotting, $48 \mathrm{~h}$ post-transfection. GAPDH was used as a loading control. The expression levels of CYLD were markedly lower in the mimics group and higher in the inhibitors group compared with in the control groups (blank or NC groups). (F) Potential target genes in the overlap of the two gene sets. (G) Dual-luciferase reporter assays using vectors encoding putative miR-197 target sites in the CYLD 3'-UTR for both wt and mut type. In the pGLO-mut-CYLD groups, no significant difference was observed between the relative luciferase activity of the cells co-transfected with the miR-197-3p mimics and cells co-transfected with NC. Conversely, the relative luciferase activity of the groups transfected with pGLO-wt-CYLD + miR-197-3p was markedly lower than in the pGLO-wt-CYLD + NC group. "P $<0.05$ vs. NC + wild-type group Normalized data were calculated as Renilla/firefly luciferase activity. CYLD, lysine 63 deubiquitinase; miR, microRNA; mut, mutant; NC, negative control; OD, optical density; UTR, untranslated region; wt, wild-type.

transfected into the human LUAD cell lines, HCC827 and Calu-3. Transfection efficiency was confirmed using qPCR (Fig. 1B and C). MTT assays were employed to investigate the effects of miR-197-3p on HCC827 and Calu-3 cell viability. Cells transfected with miR-197-3p inhibitors exhibited a decrease in proliferative ability; however, an increase in proliferative ability was observed within the miR-197-3p mimic-transfected cells compared with in the control group (Fig. 1D and E). These results indicated that the inhibition of miR-197-3p reduced the proliferative ability of LUAD cells in vitro; therefore, miR-197-3p may serve a role in the tumorigenesis of LUAD.

Inhibition of miR-197-3p enhances LUAD cell apoptosis. MTT assays were conducted to investigate the association between miR-197-3p and decreased cell proliferation. Caspase-3/7 apoptosis reagents were employed to analyze apoptosis of transfected HCC827 and Calu-3 cells. As presented in Fig. 1F and G, fluorescent activity of the miR-197-3p inhibitor-transfected group was significantly increased; however, cells transfected with miR-197-3p mimics exhibited reduced fluorescence compared with the blank and NC groups. These results indicated that downregulation of miR-197-3p enhanced LUAD cell apoptosis via caspase-3/7 activity.

miR-197-3p promotes tumorigenesis in HBE cells. miR-197-3p mimics were transfected into HBE cells to further analyze the oncogenic effects of miR-197-3p, and transfection efficiency was confirmed by RT-qPCR (Fig. 2A). In addition, the proliferative ability of transfected cells was analyzed via an MTT assay. The results of the present study demonstrated that miR-197-3p overexpression promoted HBE cell proliferation, but inhibited apoptosis; therefore, miR-197-3p may serve a role in oncogenesis (Fig. 2B and C). 
A

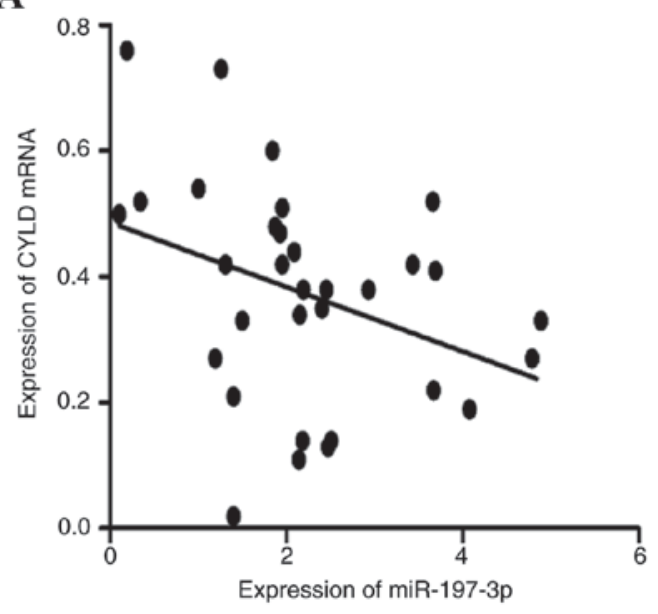

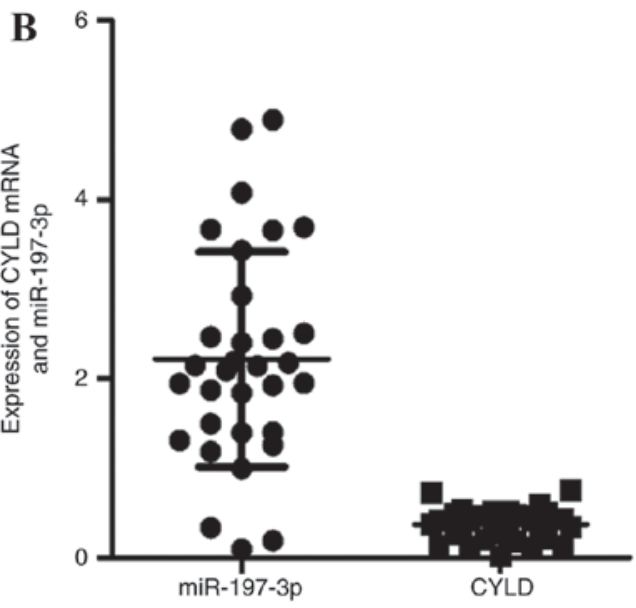

Figure 3. Association between CYLD mRNA and miR-197-3p expression within lung adenocarcinoma tissues. (A) CYLD mRNA expression levels decreased with elevated miR-197-3p expression. (B) Distribution of CYLD mRNA and miR-197-3p expression. CYLD, lysine 63 deubiquitinase; miR, microRNA.

miR-197-3p targets CYLD by binding to its 3'-UTR. To understand the observed miR-197-3p-induced tumorigenesis of LUAD cells, a bioinformatics analysis was performed using TargetScan and miRanda, after which the results were confirmed using western blotting and dual-luciferase reporter assays (Fig. 2D-G). Overlap analysis revealed 278 genes within the two gene sets. In order to narrow the scope of the predictions, genes were selected based on the total context++ score $(<-0.2)$ and total sites $(\geq 2)$ predicted by TargetScan (Fig. 2F). Among these genes, CYLD was selected, as previous studies have reported the association of CYLD downregulation with carcinogenesis (7). The potential target sites for miR-197-3p in the CYLD mRNA 3'-UTR are presented in Fig. 2D. To investigate the interaction between miR-197-3p and CYLD mRNA, CYLD protein expression levels were analyzed within HCC827 cells transfected with miR-197-3p mimics or miR-197-3p inhibitors. The results of western blotting suggested that the expression of CYLD was markedly reduced within the mimics group and elevated within the inhibitors group compared with in the control groups (blank or NC; Fig. 2E). The dual-luciferase reporter assays were conducted to confirm the results. Dual-luciferase reporter vectors containing either the mutant or wt 3'-UTR of CYLD mRNA were constructed, and co-transfected with the miR-197-3p mimics or NC in HCC827 cells. In the pGLO-mut-CYLD groups, no significant difference was observed between the relative luciferase activity of the cells co-transfected with the miR-197-3p mimics and the cells co-transfected with NC. Conversely, the relative luciferase activity of the cells co-transfected with pGLO-wt-CYLD + miR-197-3p mimics was markedly lower than that of the pGLO-wt-CYLD + NC group $(\mathrm{P}<0.05$; Fig. 2G). These results revealed that miR-197-3p interacted directly with the 3'-UTR of CYLD mRNA, inversely regulating CYLD expression.

miR-197-3p is negatively associated with CYLD mRNA expression within LUAD specimens. The association between CYLD mRNA and miR-197-3p expression within LUAD specimens is presented in Fig. 3A and B. Spearman's correlation analysis indicated that miR-197-3p was inversely associated with CYLD mRNA expression $(r=-0.436$, $\mathrm{P}<0.05)$.

CYLD is a LUAD suppressor gene. The present study investigated whether abnormal ectopic CYLD expression is sufficient to promote the proliferation of HCC827 and Calu-3 cells. The transfection efficiency of the CYLD overexpression vector was confirmed by RT-qPCR (Fig. 4A). Proliferative ability of the LUAD cells was inhibited in response to CYLD overexpression (Fig. 4B and C). In addition, CYLD overexpression markedly increased apoptosis of HCC827 and Calu-3 cells (Fig. 4D and E). These results indicated that CYLD overexpression and miR-197-3p suppression may inhibit cell proliferation and enhance apoptosis; therefore, CYLD overexpression may rescue the effects of miR-197-3p on cell function. The association between the mRNA expression levels of CYLD and LUAD prognoses of 866 patients from the Kaplan-Meier plotter database was analyzed using the Kaplan-Meier plotter database (http://kmplot.com/). The results demonstrated that the mRNA expression levels of CYLD were associated with overall survival (Fig. 5).

\section{Discussion}

Several LUAD clinical therapies are currently available; however, the 5-year survival rate of patients with LUAD has yet to be improved (11). In an effort to improve prognosis, it is necessary to develop novel efficient therapeutic strategies. Downregulation of miR-197-3p has been reported to participate in the progression and development of numerous types of cancer $(5,12,13)$. However, further investigation is required to understand the expression and biological function of miR-197-3p within LUAD0. In the present study, miR-197-3p was revealed to be overexpressed within LUAD tissues compared with adjacent noncancerous tissues. The two cells lines employed in the present study are the most common models of LUAD available. The results of the present study revealed that miR-197-3p downregulation reduced proliferative ability and enhanced apoptosis of the LUAD cell lines via caspase-3/7 activation. Conversely, upregulation of 

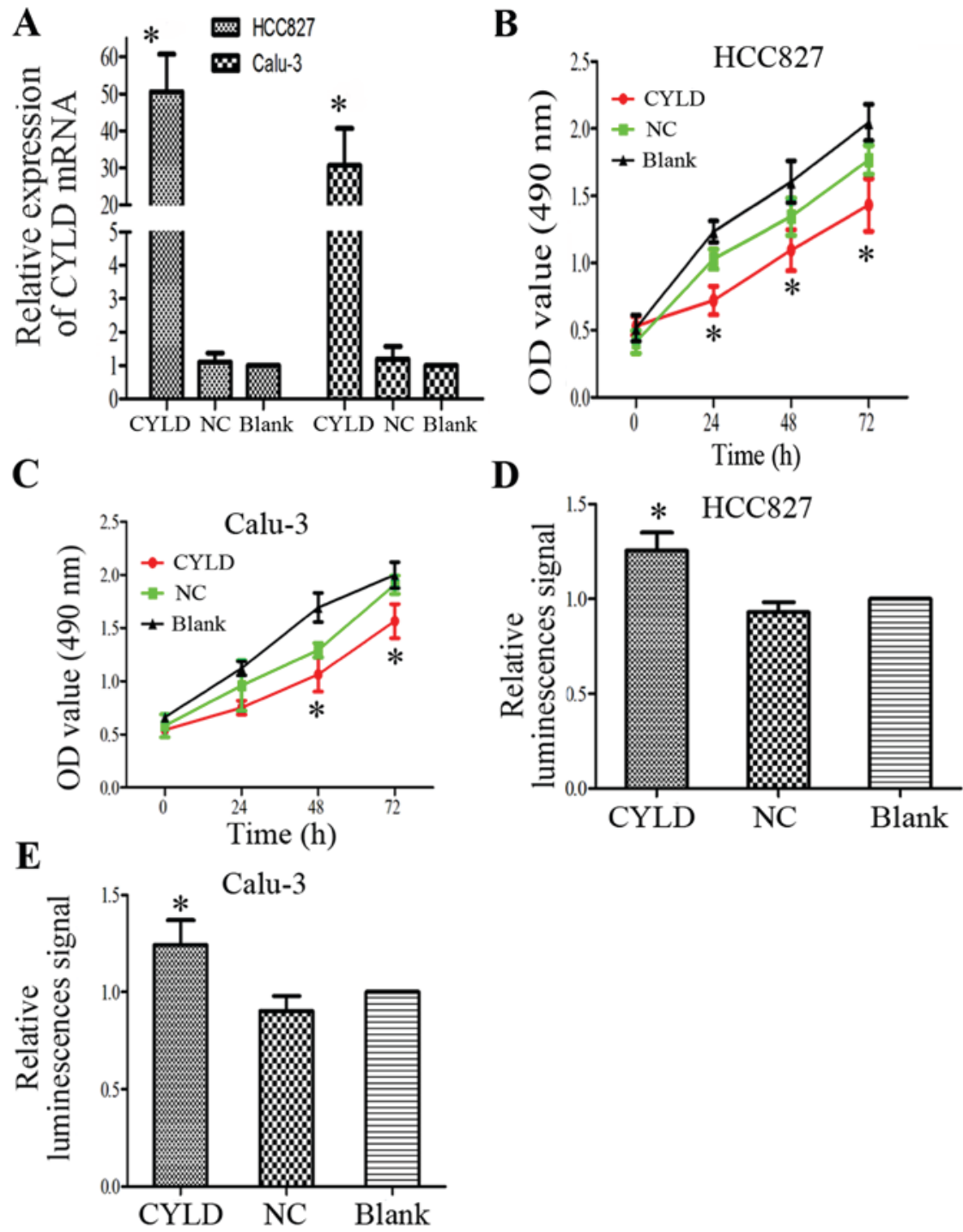

Figure 4. (A) Relative mRNA expression levels of CYLD were increased in the CYLD overexpression group compared with in the control groups. (B and C) Antineoplastic effects of CYLD overexpression. Cell proliferation was inhibited by overexpression of CYLD in HCC827 and Calu-3 cells. (D and E) Caspase activity was significantly increased by CYLD overexpression within HCC827 and Calu-3 cells. "P<0.05 vs. blank group. CYLD, lysine 63 deubiquitinase; miR, microRNA; NC, negative control; OD, optical density.

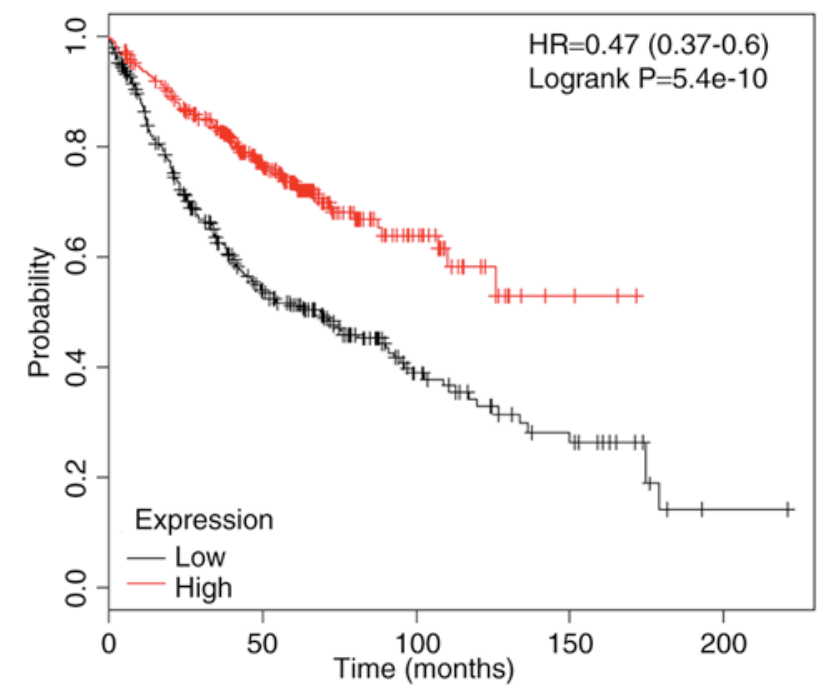

Figure 5. Kaplan-Meier plot downloaded from the Kaplan-Meier plotter database. Elevated lysine 63 deubiquitinase expression was associated with an improved prognosis within patients with lung adenocarcinoma.
miR-197-3p promoted HBE cell tumorigenesis, thus suggesting that miR-197-3p exerts oncogenic effects. A bioinformatics analysis indicated that two miR-197-3p binding sites are present within the 3'UTR of CYLD mRNA. This prediction was applied to investigate the regulatory role of miR-197-3p on proliferation and apoptosis. Subsequently, luciferase reporter assays were conducted; the luciferase activity exhibited by the pGLO-wt-CYLD group was reduced in response to transfection with miR-197-3p mimics, thus providing confirmation that CYLD mRNA is a target of miR-197-3p. In the present study, cells were transfected with a CYLD overexpression vector, and its role as a tumor suppressor gene was confirmed. The results of the present study indicated that miR-197-3p downregulation may partly inhibit LUAD cell proliferation via CYLD upregulation, thus resulting in inhibition of LUAD progression.

CYLD is a crucial enzyme involved in deubiquitination. CYLD has been reported to regulate numerous signaling pathways, including transforming growth factor (TGF)- $\beta$, 
Wnt $/ \beta$-catenin and nuclear factor $(\mathrm{NF})-\kappa \mathrm{B}$ signaling, and therefore affects tumorigenesis (14-17). Furthermore, the downregulation of CYLD has been confirmed within various malignant tumors (18-21). In the present study, the data suggested that aberrant expression of CYLD may participate in the progression and development of LUAD, and CYLD may serve as a prognostic predictor of LUAD.

The main limitation of the present study is that a relatively small number of patients were involved in the analysis; in addition, detailed information for these individuals was not collected. In addition, in vivo assays and in-depth investigation into the mechanisms underlying the effects of CYLD and miR-197-3p are also required. Therefore, further studies are essential to investigate the regulation of TGF- $\beta$, Wnt and $\mathrm{NF}-\kappa \mathrm{B}$ signaling pathways by miR-197-3p in the future. In conclusion, the findings of the present study indicated that miR-197-3p regulated the biological behaviors of LUAD via CYLD downregulation. In addition, the expression of CYLD may be significantly associated with the prognosis of LUAD. These conclusions suggested that the miR-197-3p/CYLD interaction may be applied in the development of novel LUAD-targeted treatments.

\section{Acknowledgements}

The present study was supported by the Liaoning Province Natural Science Foundation (grant no. 2013021041).

\section{References}

1. Miller KD, Siegel RL, Lin CC, Mariotto AB, Kramer JL, Rowland JH, Stein KD, Alteri R and Jemal A: Cancer treatment and survivorship statistics, 2016. CA Cancer J Clin 66: 271-289, 2016.

2. Diaz-Garcia CV, Agudo-López A, Perez C, López-Martín JA, Rodríguez-Peralto JL, de Castro J, Cortijo A, Martínez-Villanueva M, Iglesias L, García-Carbonero R, et al: DICER1, DROSHA and miRNAs in patients with non-small cell lung cancer: Implications for outcomes and histologic classification. Carcinogenesis 34: 1031-1038, 2013.

3. Lau NC, Lim LP, Weinstein EG and Bartel DP: An abundant class of tiny RNAs with probable regulatory roles in Caenorhabditis elegans. Science 294: 858-862, 2001.

4. Yanaihara N, Caplen N, Bowman E, Seike M, Kumamoto K, Yi M, Stephens RM, Okamoto A, Yokota J, Tanaka T, et al: Unique microRNA molecular profiles in lung cancer diagnosis and prognosis. Cancer Cell 9: 189-198, 2006.

5. Fiori ME, Barbini C, Haas TL, Marroncelli N, Patrizii M, Biffoni M and De Maria R: Antitumor effect of miR-197 targeting in p53 wild-type lung cancer. Cell Death Differ 21: 774-782, 2014.

6. Bignell GR, Warren W, Seal S, Takahashi M, Rapley E, Barfoot R, Green H, Brown C, Biggs PJ, Lakhani SR, et al: Identification of the familial cylindromatosis tumour-suppressor gene. Nat Genet 25: 160-165, 2000.
7. Kovalenko A, Chable-Bessia C, Cantarella G, Israel A, Wallach D and Courtois G: The tumour suppressor CYLD negatively regulates NF-kappaB signalling by deubiquitination. Nature 424: 801-805, 2003.

8. Lin $\mathrm{X}$, Chen $\mathrm{Q}$, Huang $\mathrm{C}$ and $\mathrm{Xu} \mathrm{X}$ : CYLD promotes TNF- $\alpha$-Induced cell necrosis mediated by RIP-1 in human lung cancer cells. Mediators Inflamm 2016: 1542786, 2016.

9. Wang YY, Wu ZY, Wang GC, Liu K, Niu XB, Gu S and Meng JS: LINC00312 inhibits the migration and invasion of bladder cancer cells by targeting miR-197-3p. Tumour Biol 37: 14553-14563, 2016.

10. Livak KJ and Schmittgen TD: Analysis of relative gene expression data using real-time quantitative PCR and the 2(-Delta Delta C(T)) method. Methods 25: 402-408, 2001.

11. Travis WD: Pathology of lung cancer. Clin Chest Med 32: 669-692, 2011.

12. Wang YY, Wu ZY, Wang GC, Liu K, Niu XB, Gu S and Meng JS: LINC00312 inhibits the migration and invasion of bladder cancer cells by targeting miR-197-3p. Tumour Biol 37: 14553-14563, 2016.

13. Chen X, Xu Y, Cao X, Chen Y, Jiang J and Wang K: Associations of Il-1 Family-Related polymorphisms with gastric cancer risk and the role of Mir-197 In Il-1f5 Expression. Medicine (Baltimore) 94: e1982, 2015.

14. Lim JH, Jono H, Komatsu K, Woo CH, Lee J, Miyata M, Matsuno T, Xu X, Huang Y, Zhang W, et al: CYLD negatively regulates transforming growth factor- $\beta$-signalling via deubiquitinating Akt. Nat Commun 3: 771, 2012.

15. Tauriello DV, Haegebarth A, Kuper I, Edelmann MJ, Henraat M, Canninga-van Dijk MR, Kessler BM, Clevers H and Maurice MM: Loss of the tumor suppressor CYLD enhances Wnt/beta-catenin signaling through K63-linked ubiquitination of Dvl. Mol Cell 37: 607-619, 2010.

16. Pannem RR, Dorn C, Ahlqvist K, Bosserhoff AK, Hellerbrand C and Massoumi R: CYLD controls c-MYC expression through the JNK-dependent signaling pathway in hepatocellular carcinoma. Carcinogenesis 35: 461-468, 2014.

17. Wang WY, Lim JH and Li JD: Synergistic and feedback signaling mechanisms in the regulation of inflammation in respiratory infections. Cell Mol Immunol 9: 131-135, 2012.

18. Gautheron $\mathbf{J}$ and Luedde T: A novel player in inflammation and cancer: The deubiquitinase CYLD controls HCC development. J Hepatol 57: 937-939, 2012.

19. Font-Burgada J, Seki E and Karin M: CYLD and HCC: When being too sensitive to your dirty neighbors results in self-destruction. Cancer Cell 21: 711-712, 2012.

20. Urbanik T, Köhler BC, Boger RJ, Wörns MA, Heeger S, Otto G, Hövelmeyer N, Galle PR, Schuchmann M, Waisman A and Schulze-Bergkamen H: Down-regulation of CYLD as a trigger for NF- $\mathrm{KB}$ activation and a mechanism of apoptotic resistance in hepatocellular carcinoma cells. Int $\mathrm{J}$ Oncol 38: 121-131, 2011.

21. Hayashi M, Jono H, Shinriki S, Nakamura T, Guo J, Sueta A, Tomiguchi M, Fujiwara S, Yamamoto-Ibusuki M, Murakami K, et al: Clinical significance of CYLD downregulation in breast cancer. Breast Cancer Res Treat 143: 447-457, 2014.

This work is licensed under a Creative Commons Attribution-NonCommercial-NoDerivatives 4.0 International (CC BY-NC-ND 4.0) License. 